\title{
Modeling of Liquid Hot Metal Sloshing in Ladles During Transportation by Locomotives (A Bond Graph Method)
}

\author{
Abhijit Roy, Anup Kumar Saha
}

\begin{abstract}
Safe and secure transportation of liquid hot metal in steel plants is very challenging. About ninety percent of transportation is by means of locomotives. Sloshing is a common phenomenon in open container liquid transportation due to external excitation. Non-linear sloshing dynamics of liquid hot metal in ladle due to locomotive movement is the prime consideration of this paper. Liquid hot metal inside the ladle has been considered in the line of an equivalent mechanical system. Resulting forces and moments acting on the ladle inside wall are considered equal in all senses. An equivalent mechanical dynamic system representation of sloshing by bond graph modellinghas been formulated by keeping records in a satisfactory way.Future research scopes has been identifiedin parallel with an outline mapped. Hot metal liquid has two distinct components of the hydrodynamic pressure in consideration of rigid containershas been identified. Bottom segment of the molten metal column moves unison with the ladleandis directlyproportional with the acceleration of the ladle. Whereas the second one 'convective' pressure at the free surface, particularly experiences the sloshing tendency due to external forces.
\end{abstract}

Keywords: Bond graph Dynamics Locomotives Modeling Simulation Sloshing

\section{INTRODUCTION}

Ladle car as shown in Fig 1 is a wagon like structure on which there is a seat to rest the ladle [1]. The wheels rolls over the rail while pushed or pulled by locomotives. The buffer or stopper (that contains spring ) of ladle car and locomotives while comes in contact, external excitation comes into play. This excitation transmitted to the Ladle body, refractory lining and ultimately liquid hotmetal inside the ladle. In rigid containers of hot liquidmetal, has two distinct components of the hydrodynamic pressure. Bottom segment of the molten metal column moves unison with the ladle and is in direct proportion with the acceleration of the locomotives. Whereas the second one at the free surface, also known to be "convective" pressure, particularly experiences the sloshing tendency due to external forces.

Momentum of locomotive, sudden brake or torque and joint in rail lines which are the causes of different external forces [6] that may cause sloshing e.g. due to [7]. The liquid hot metal dynamics inside the ladle can be approximated by a very close resemblance to mechanical system [8]. For the urpose of resemblance the assumption of equal resultant forces and that of the moments acting on the ladle wall is taken into consideration [9].

\section{Mathematical Modelling}

An equivalent mechanical model as shown in Fig.2 consisting of a rigid mass moving in unison with the ladle, and a series of masses $M_{n}$, representing the equivalent mass of each sloshing mode. Stiffness $K_{n}$ and damping $R_{n}$ represents for each modal mass [3]. Two modal masses have been considered for simplicity.

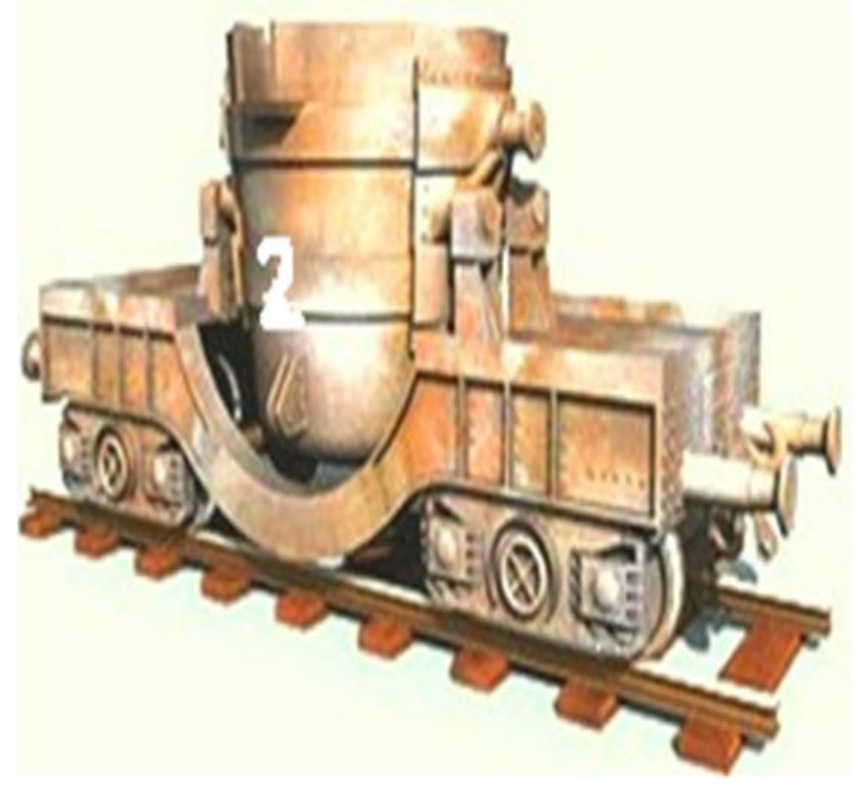

Fig. 1 Ladle Car ready for transportation of Technology Durgapur, Durgapur -713209, India

Anup Kumar Saha, Department of Mechanical Engineering, National Institute of Technology Durgapur, Durgapur -713209, India 


\begin{tabular}{|c|c|}
\hline \multicolumn{2}{|c|}{ Nomenclature } \\
\hline $\mathrm{P}$ & density of liquid metal \\
\hline $\mathrm{U}$ & velocity vector in $\mathrm{x}$ direction \\
\hline $\mathrm{V}$ & velocity vector in y direction \\
\hline $\mathrm{W}$ & velocity vector in $\mathrm{z}$ direction \\
\hline$\Phi$ & arbitrary conserved property of moving fluid particle \\
\hline Q & generalized coordinates \\
\hline Q & generalized forces \\
\hline $\mathrm{I}_{0}$ & moment of inertia of total rigid mass \\
\hline $\mathrm{I}_{\mathrm{F}}$ & $\begin{array}{l}\text { mass moment of inertia of the liquid metal about } \\
\text { they-axis and passing through the centre of mass }\end{array}$ \\
\hline $\mathrm{L}$ & Lagrangian \\
\hline $\mathrm{T}$ & kinetic energy \\
\hline $\mathrm{U}$ & potential energy \\
\hline$\Lambda$ & dissipation energy \\
\hline$\xi_{1 \mathrm{n}}$ & roots of determinants \\
\hline $\mathrm{M}_{0}$ & rigid mass moving unison with the ladle \\
\hline $\mathrm{M}_{\mathrm{n}}$ & modal mass of $\mathrm{n}^{\text {th }}$ number \\
\hline $\mathrm{K}_{\mathrm{n}}$ & spring stiffness of each modal masses \\
\hline $\mathrm{R}_{\mathrm{n}}$ & damping coefficient of modal masses \\
\hline $\mathrm{H}_{0}$ & depth of rigid mass from CG \\
\hline $\mathrm{H}_{\mathrm{n}}$ & height of modal mass from CG \\
\hline $\mathrm{M}_{\mathrm{y}}$ & Pitching moment caused by hydrodynamic force \\
\hline$\dot{x}_{0}$ & velocity of rigid bottom liquid metal with ladle mass \\
\hline$\dot{x}_{\mathrm{n}}$ & velocity of each modal mass \\
\hline$\ddot{x}_{0}$ & acceleration of rigid bottom liquid metal with ladle mass \\
\hline$\ddot{x}_{\mathrm{n}}$ & acceleration of each modal mass \\
\hline $\mathrm{F}$ & hydrodynamic force \\
\hline$\Omega$ & angular frequency of oscillation \\
\hline$\omega_{\mathrm{n}}$ & angular frequency of $\mathrm{n}^{\text {th }}$ mode of oscillation \\
\hline$\beta_{\mathrm{n}}$ & damping factor of equivalent dashpot \\
\hline$\Omega$ & frequency of external excitation \\
\hline $\mathrm{A}_{0}$ & Integral constant \\
\hline$\varphi_{0}$ & $\begin{array}{l}\text { rotational motion of the container about the } \mathrm{z} \text {-axis } \\
\text { through the center of gravity }\end{array}$ \\
\hline $\mathrm{R}$ & radius of the ladle \\
\hline $\mathrm{x}_{\mathrm{n}}$ & $\begin{array}{l}\text { displacement of the equivalent mass relative to the } \\
\text { container wall }\end{array}$ \\
\hline $\mathrm{X}_{0}$ & displacement of the container \\
\hline
\end{tabular}

Fluid total mass:

$$
M_{F}=M_{o}+\sum_{n=1}^{\infty} M_{n}
$$

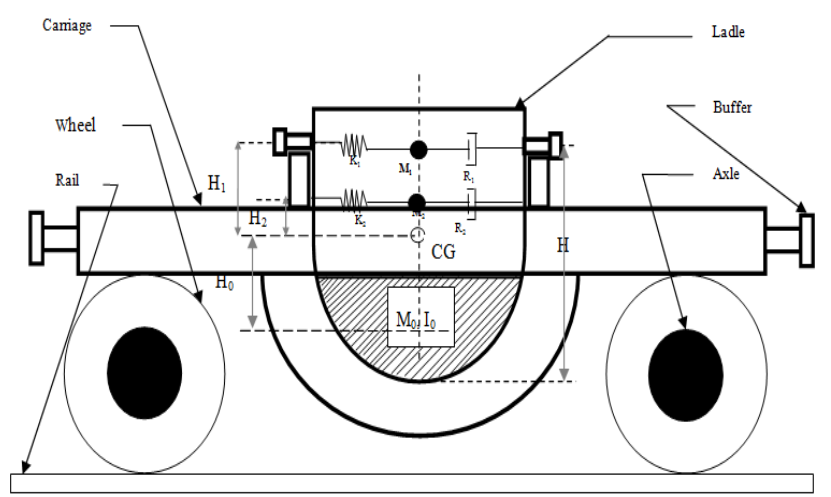

Fig. 2. Equivalent Mechanical Model of Ladle on Ladle Car. Mass moment of inertia of the liquid metal about the $\mathrm{x}$-axis and passing through the centre of mass of the molten metal:

$I_{F}=I_{o}+M_{0} H_{0}{ }^{2}+\sum_{n=1}^{\infty} M_{n} H_{n}{ }^{2}$

Preservation of Centre of mass should be as per following equation:

$M_{0} H_{0}-\sum_{n=1}^{\infty} M_{n} H_{n}=0$

The equations of motion of the equivalent model developed using Lagrange's equation may be derived as follows:

$$
\frac{d}{d t}\left(\frac{\delta}{\delta \dot{p}} L\right)-\left(\frac{\delta}{\delta p} L\right)=-\frac{\delta}{\delta \dot{q}} \lambda+Q
$$

Where the nomenclatures are, Lagrangian $\mathrm{L}=\mathrm{T}-\mathrm{U}, \mathrm{q}$ is generalized coordinates, $Q$ is generalized forces and $\lambda$ is dissipation energy function. $\mathrm{T}$ and $\mathrm{U}$ are the kinetic and potential energies, respectively.

Kinematic Energy:

$T=-\frac{1}{2} M_{0}\left(\dot{x}_{0}-H_{0} \dot{\phi}\right)^{2}+\frac{1}{2} I_{0} \dot{\phi}^{2}+\frac{1}{2} \sum_{n=1}^{\infty} M_{n}\left(\dot{x}_{n}+\dot{x}+H_{n} \phi\right)^{2}$

Potential Energy:

$$
U=\frac{1}{2} M_{0} H_{0} g \phi^{2}-\frac{1}{2} g \phi^{2} \sum M_{n} H_{n}-g \phi \sum_{n=1}^{\infty} M_{n} x_{n}+\frac{1}{2} \sum_{n=1}^{\infty} K_{n} x_{n}^{2}
$$

Dissipation energy:

$$
\lambda=\frac{1}{2} \sum_{n=1}^{\infty} R_{n} \dot{x}_{n}^{2}=\sum_{n=1}^{\infty} M_{n} \omega_{n} \beta_{n} \dot{x}_{n}^{2}
$$

The generalised coordinate $\{q\}$ and force $\{Q\}$ are represented by vectors 


$$
\{q\}=\left\{\begin{array}{lll}
x & x_{n} & \phi
\end{array}\right\}^{\mathrm{T}} \quad\{0\}=\left\{\begin{array}{lll}
-F_{x} & 0 & M_{y}
\end{array}\right\}^{\mathrm{T}}
$$

Now applying Lagrange's from equation (4) and using expressions (5) to (8) following equations of motions can be derived. Force equation will be $M_{0}\left(\ddot{x}-H_{0} \ddot{\phi}\right)+\sum_{n=1}\left[M_{n}\left(\ddot{x}+\ddot{x}_{n}+H_{n} \ddot{\phi}\right)\right]=-F_{x}$

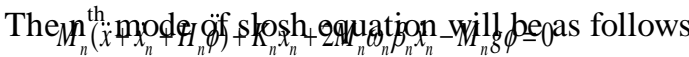

Whereas moment equation becomes

$$
I_{0} \ddot{\phi}+M_{0} H_{0}\left(\ddot{x}-H_{0} \ddot{\phi}\right)-g \sum_{n=1} M_{n} x_{n}+\sum_{n=1}\left[M_{n} H_{n}\left(\ddot{x}+\ddot{x}_{n}+H_{n} \ddot{\phi}\right)\right]=M_{y}
$$

Equations (9) to (11) fully describe the translation and pitching of the model. Now let us derive the equation of lateral excitation for combined sway and pitching of the damped model

$$
M_{n} \ddot{x}_{n}(t)+R_{n} \dot{x}_{n}(t)+K_{n} x_{n}(t)=M_{n} \Omega^{2} A_{0} \sin \Omega t+M_{n}\left(g+H_{n} \Omega^{2}\right) \phi \sin \Omega t
$$

Liquid metal at the hemispherical bottom of ladle do not participate in sloshing and mass of this portion is unison with ladle mass (as mentioned earlier),sodiscussion here covered with cylindrical portion only and general solution for a cylindrical shaped ladle will be as follows:

Due to ladle car movement the following hydrodynamic force with combination of translational and pitching excitations (of liquid inside ladle):

$F_{x}=M_{F} A_{0} \Omega^{2} \sin \Omega t\left\{1+\sum_{n=1}^{\infty} \frac{2 R \Omega^{2} \tanh \left(\xi_{\mathrm{ln}} H / R\right)}{\xi_{\mathrm{m}} H\left(\xi_{\mathrm{m}}^{2}-1\right)\left(\omega_{n}^{2}-\Omega^{2}\right)}\right\}+M_{F} \phi \Omega^{2} \sin \phi t \times \sum_{n=1}^{\infty} 2 R \tanh \left(\xi_{\ln } H / R\right) \Omega^{2}\left[(H / 2) \Omega^{2}\right.$

$$
\left\{\frac{\left.\left(1-\left(4 R / \xi_{\mathrm{m}} H\right) \tanh \left(\xi_{\mathrm{m}} H / R\right)+g\right)\right]}{\xi_{\mathrm{m}} H\left(\xi_{\mathrm{m}}^{2}-1\right)\left(\omega_{n}^{2}-\Omega^{2}\right)}\right\}
$$

Ritehingimoment caused by hydrodynamic force is

$$
\begin{aligned}
& \left\{\begin{array}{c}
I_{F}+m_{F} \sum_{n=1}^{\infty} \frac{2 R H^{2} \Omega^{2} \tanh \left(\xi_{\mathrm{ln}} H / R\right)}{\xi_{\ln } H\left(\xi_{\mathrm{ln}}^{2}-1\right)} \frac{\left[\left(1-4 R / \xi_{\mathrm{ln}} H\right) \tanh \left(\xi_{\mathrm{ln}} H / R\right)\right]^{2}}{4\left(\omega_{n}^{2}-\Omega^{2}\right)}+ \\
M_{F} \sum_{n=1}^{\infty} \frac{2 R \tanh \left(\xi_{\mathrm{ln}} H / R\right)}{\xi_{\ln } H\left(\xi_{\mathrm{ln}}^{2}-1\right)}\left\{\frac{(g / \Omega)^{2}+g h\left[1-\left(4 R / \xi_{\mathrm{ln}} H\right) \tanh \left(\xi_{\mathrm{ln}} H / R\right)\right]}{\left(\omega_{n}^{2}-\Omega^{2}\right)}\right\}
\end{array}\right\} \\
& +M_{F} A_{0} \Omega^{2} \sin \Omega t \times M_{F} \sum_{n=1}^{\infty} \frac{2 R \tanh \left(\xi_{\mathrm{n}} H / R\right)}{\xi_{\ln } H\left(\xi_{\mathrm{m}}^{2}-1\right)}\left\{\frac{g+\left(h \Omega^{2} / 2\right)\left[1-\left(4 R / \xi_{\mathrm{ln}} H\right) \tanh \left(\xi_{\mathrm{m}} H / R\right)\right]}{\left(\omega_{n}^{2}-\Omega^{2}\right)}\right\}
\end{aligned}
$$

Now we can derive the Moment of inertia from above three equations as follows:

$\mathrm{I}_{\mathrm{F}}=\mathrm{I}_{0}+\mathrm{M}_{0} \mathrm{H}_{\mathrm{o}}^{2}+\sum_{\mathrm{n}=1}^{\infty} \mathrm{M}_{\mathrm{n}} \mathrm{H}_{\mathrm{n}}^{2}=\mathrm{M}_{\mathrm{F}}\left\{\frac{H^{2}}{12}+\frac{R^{2}}{4}-8 R^{2} \sum_{n=1}^{\infty} \frac{1-\left(2 R / \xi_{\mathrm{m}} H\right) \tanh \left(\xi_{\mathrm{H}} H / 2 R\right)}{\xi_{\mathrm{n}}\left(\xi_{\mathrm{m}}^{2}-1\right)}\right\}$

\section{BONDGRAPH MODELING:}

Here the oscillation of liquid metal inside the ladle along $\mathrm{x}$-direction acts as a spring -dashpot combination, while ladle pushed or pulled by locomotives. Due to external excitation applied at buffernear the C.G. of the ladle the oscillation along $\mathrm{X}$-axis is to a large extent, causing sloshing in $\mathrm{x}$-direction i.e. Sway motion in $\mathrm{x}$-direction. In the following Fig. 3 a bond graph model of liquid metal sloshing in ladle while transported by locomotive is shown.

Bond graph model generated with only two mode of slosh taken into consideration while liquid metal sloshes due to external excitation during transportation. There are mainly two part, the upper part which is bond graph model of the ladle car with ladle, resembles with popular bond graph model of a car body while the lower part thetwo mode of sloshing of liquid hot metal. In upper part it can be seenC (19, (12)i.e. $\mathrm{Kf}, \mathrm{Kr}$ are representing stiffness of front and rear wheel of ladle car and for damping of same isR $(20,23)$ i.e. $\mathrm{Rf}$ and Rr. SF25 and SF27 representing the rail reaction on front and rear wheels.I30 representing rotary inertia

and I3 representing mass of ladle and ladle car. The $1_{\dot{z}}$ represents linear junction and $1_{\dot{\theta} 1}$ rotary junctions respectively of the ladle car and flow from $1_{\dot{z}}$ junction meet $1_{\dot{x}}$ junction, whichrepresents the velocity of the liquid inside the ladle in $\mathrm{x}$ - direction. A transformer connected between $1_{\dot{z}}$ junction and $1_{\dot{x}}$ junction through two 0 -junctions converts linear motion from $\mathrm{z}$-direction to $\mathrm{x}$-direction and modulus of it is derived from activated bonds C6 and C17. Now flow from transformer (TF) transmitted through 0 -junction to $1_{\dot{x}}$ junction. The rotary inertia connected to $1_{\dot{\theta} 1}$ junction denoted by I (30).The inertia (I) at this 0 -junction though bond number 14 representing the mass of ladle along with liquid metal at bottom of ladle that doesn't participate in sloshing. As the simulation of the model is done with two modal masses, stiffness and damping property of first and second modal masses are represented by $\mathrm{K} 1(38), \mathrm{K} 2(42)$ andR1(39), R2(42) whereas I1 (39) and I2 (44) are respective of 1 stand 2 ndmodal masses respectively. Two flow activated $\mathrm{C}$ bonds numbered 37 and 40 attaches at 1 junctions are the observation points to find the sloshing effect or deflection of the two modal masses respectively due to external excitation on ladle by locomotives represented by SF 12 .

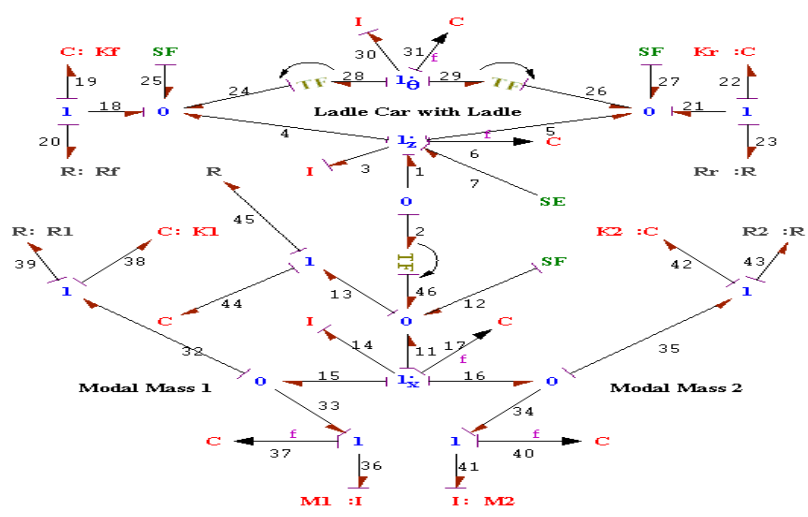

Fig.3. Bondgraph Model of hotmetal liquid slosh due to external excitation by locomotive. 


\section{RESULTS AND DiscuSSION}

Simulation results obtained with parametric values as given in table 1 below.From the first graphs generated after simulation it can be seen in Figure 4 and Figure 5 that maximum velocity attained by first and second modal masses are $8.21 \mathrm{e}+0 \mathrm{~m} / \mathrm{s}$ and $7.68 \mathrm{e}+0 \mathrm{~m} / \mathrm{s}$ at 2.13623 th and 2.1416 th second respectively of simulation run for 200 seconds. Whereas simulation velocities are minimum for first as well as second modal masses which are $3.52 \mathrm{e}+0 \mathrm{~m} / \mathrm{s}$ and $3.29 \mathrm{e}+0 \mathrm{~m} / \mathrm{s}$ respectively when speed of locomotive decreased at two times to that of previous one.Directions of velocity of both modal masses are opposite to that of locomotive movement. Sloshing responsepattern is similar to that numerically obtained by Patron and experimentally by Werner et al [7].

Table 1.Parametric Values.

\begin{tabular}{ll}
\hline Description & \multicolumn{1}{c}{ Values } \\
Mass of the Ladle & $150 \times 10^{3} \mathrm{Kg}$ \\
Stiffness of $1^{\text {st }}$ modal mass & $180000 \mathrm{~N}$ \\
Stiffness of $2^{\text {nd }}$ modal mass & $150000 \mathrm{~N}$ \\
Damping of $1^{\text {st }}$ modal mass & $900 \mathrm{Ns} / \mathrm{m}$ \\
Damping of $2^{\text {nd }}$ modal mass & $1000 \mathrm{Ns} / \mathrm{m}$ \\
Stiffness of Ladle car structure & $5.4 \times 10^{7} \mathrm{~N}$ \\
Damping of Ladle car structure & $5.4 \times 10^{5} \mathrm{~N}$ \\
Stiffness of ladle car wheel & $5 \times 10^{7} \mathrm{Ns} / \mathrm{m}$ \\
Damping of ladle car wheel & $5 \times 10^{5} \mathrm{Kg}-\mathrm{m}$ \\
Moment of inertia of ladle car & $50000 \mathrm{Kg}$ \\
Mass of $1^{\text {st }}$ modal mass & $30000 \mathrm{Kg}$ \\
Mass of $2^{\text {nd }}$ modal mass & $20000 \mathrm{~m}$ \\
Step-up height & $0.06 \mathrm{~m}$ \\
Step-up length & $0.2 \mathrm{~m}$ \\
Velocity of the locomotive & $5.55 \mathrm{~m} / \mathrm{s}$ \\
Distance between two wheels & $4 \mathrm{~m}$ \\
\hline
\end{tabular}

The shape of the graph generated to find the deflection of ladle car CG as in figure 6 is $4.72 \mathrm{e}-2 \mathrm{~m}$ and $-2.72 \mathrm{e}-2 \mathrm{~m}$ in both direction. The maximum velocity attained bythe $\mathrm{CG}$ of liquid metal in ladle is $-5.97 \mathrm{e}+0 \mathrm{~m} / \mathrm{s}$. Parameters require to be changed as per optimum design needed.
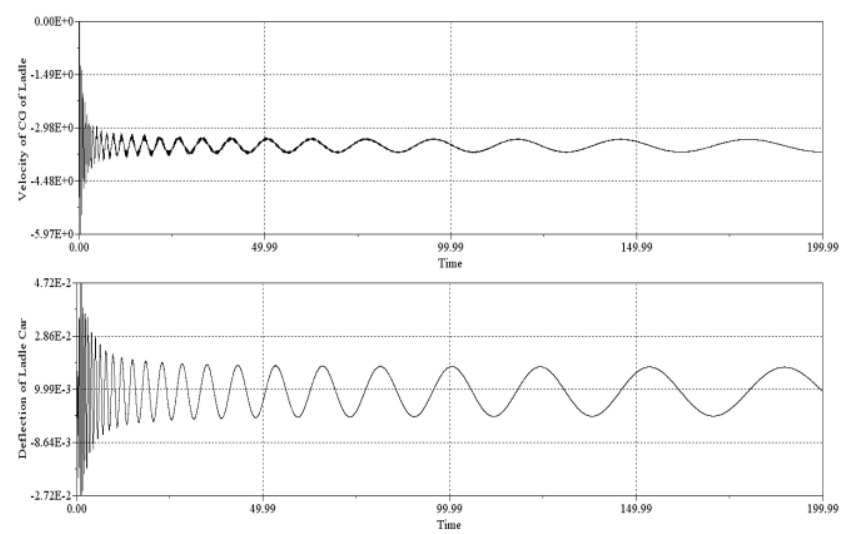

\section{REFERENCES}

[1] RanjitKarmakar, A.Mukherjee, Dynamics of Electric Overhead Travelling Cranes, Mech.Mach.Theory 25 (1990) 29-39.

[2] Quang Hieu Ngo, Keum-Shik Hong, Il HyoJung, Adaptive control of an axially moving system, Journal of Mechanical Science and Technology 23 (2009)3071-3078.

[3] Abhijit Roy, A.K. Saha, Bond Graph Modeling and Simulation of Liquid Metal Sloshing in Ladle, International Conference on Bond Graph Modeling and Simulation, ICBN 978-1-5108-2425-6 (2016) 191-197.

[4] R.A. Ibrahim, V.N. Pilipchuck, T. Ikeda, Recent Advances In Liquid Sloshing Dynamics, Applied Mechanics Reviews54 (2001) 133-199.

[5] Sir G. G. Stokes, Discussion of a Differential Equation Relating to Breaking of Railway Bridges, Mathematics Physics Paper2 (1849) 179.

[6] E. Lightfoot, Shock loading effects in overhead travelling cranes-review of dynamic stress allowances. Engng 181 (1956) 169.

[7] R.A. Ibrahim, Liquid Sloshing Dynamics, Cambridge University Press, New York, ISBN-13 978-0-5II-12492-I,Ch.5 Equivalent Mechanical models (2005) 296-334.

[8] Bamdad Mahdi, Taheri Farzin, Abtahi Niloofar, Dynamic analysis of a Hybrid Cable-Suspended Planar Manipulator, IEEE International Conference on Robotics and Automation (ICRA) (2005) 1621-1626, Washington State Convention Center Seattle, Washington, May 26-30, 2015.

[9] A.Mukherjee, R.Karmakar, A.K. Samanta Roy, Modeling and Simulation of EngineeringSystems, ISBN 81-88237-96-5 Publisher: I.K.International Publishing House Pvt Ltd.2006.

[10] Cao Lingzhi, Lu Liping, Cui Guangzhao, Wei Shangbei, Lou Feipeng, Miao Weipu, Sequential modeling and control of the dynamic load in crane lifter, pp 4844-48482009 Chinese Control and Decision Conference, 2009.

[11] T.X. Wu, D.J. Thompson,Vibration Analysis of Railway Track with Multiple wheels on the Rail,Journal of Sound and Vibration239 (2001) $69-97$.

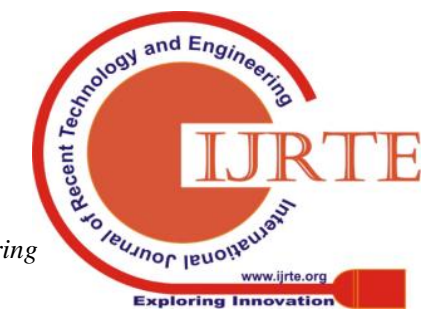

